\title{
Optimal Scheme for Estimating a Pure Qubit State via Local Measurements
}

\author{
E. Bagan, M. Baig, and R. Muñoz-Tapia \\ Grup de Física Teòrica \& IFAE, Facultat de Ciències, Edifici Cn, Universitat Autònoma de Barcelona, \\ 08193 Bellaterra (Barcelona), Spain \\ (Received 14 May 2002; published 19 December 2002)
}

\begin{abstract}
We present the optimal scheme for estimating a pure qubit state by means of local measurements on $N$ identical copies. We give explicit examples for low $N$. For large $N$, we show that the fidelity saturates the collective measurement bound up to order $1 / N$. When the signal state lays on a meridian of the Bloch sphere, we show that this can be achieved without classical communication.
\end{abstract}

DOI: $10.1103 /$ PhysRevLett.89.277904

PACS numbers: 03.67.Hk, 03.65.Ta

State estimation is a central topic in quantum mechanics. Quantum measurements give only partial information about the state of the system under consideration. If an unlimited number of copies of a given state were available, one could in principle determine exactly this state, provided an infinite number of measurements were performed. In the real world, we have access to a limited number of copies and time for a finite number of measurements, and the best we can achieve is an estimate of the state. It is, thus, of great importance to design optimal strategies which maximize the knowledge one can acquire about a general quantum state.

Over the past few years, a great deal of work has been devoted to the optimal estimation of states and many important features have been recognized [1-6], one of the most interesting ones being that collective measurements (CM) are more informative than individual ones [1,2]. The experimental implementation of such measurements seems, though, quite involved. In practice, individual von Neumann measurements are far easier to perform. Moreover, one can show that optimal individual measurements are of this type in the situations considered in this Letter [7]. In the most general local framework one is allowed to design each individual measurement according to the outcome of the previous ones. One can further assume that the copies of the system may have never interacted in the past and may be macroscopically separated. We call this a LOCC (local operations and classical communication) scheme because classical communication is essential in this situation. This is in contradistinction to collective measurements, for which the role of classical communication is played by the quantum entanglement of the measuring devices. In the LOCC context, some numerical simulations and experimental tests have been performed recently $[8,9]$.

In this Letter, we will deal with pure states of a single qubit $|\psi\rangle$. To each one of these states it corresponds a unique unit vector $\vec{n}$ on the Bloch sphere; i.e., $|\psi\rangle\langle\psi|=$ $(1+\vec{n} \cdot \vec{\sigma}) / 2$, where $\vec{\sigma}$ are the usual Pauli matrices. We will focus on two situations to which we will simply refer as $3 \mathrm{D}$ and $2 \mathrm{D}$. In the former, no prior knowledge of $\vec{n}$ is assumed whereas, for the latter (2D), $\vec{n}$ is known to lay on a meridian of the Bloch sphere. Our aim is to design LOCC measurements such that we can obtain the best estimate of $\vec{n}$ and, hence, of $|\psi\rangle$. We will investigate how good these LOCC measurements are as compared to the collective ones. For the latter, the mean fidelity $F$ is commonly used as a figure of merit and many results can be found in the literature - in particular, the large $N$ asymptotic behavior of $F$ is now known for the most interesting approaches $[2,4]$.

For $N$ identical copies of $|\psi\rangle$ optimal CM lead to a fidelity that behaves as $F_{\mathrm{CM}} \approx 1-1 / N$ for large $N$ in 3D [2]. From [10,11], one can likewise compute the asymptotic fidelity in $2 \mathrm{D}$, which is $F_{\mathrm{CM}} \approx 1-1 /(4 N)$. These results are the absolute upper bound for any measurement scheme; therefore within the LOCC framework the fidelity $F$ cannot approach unity at a rate larger than $1-1 /$ $(4 N)$ in $2 \mathrm{D}(1-1 / N$ in $3 \mathrm{D})$ [5]. In this Letter, we demonstrate that, rather unexpectedly, this asymptotic behavior can be achieved in 2D with just local measurements and no classical communication. In 3D, classical communication seems necessary to saturate the asymptotic $\mathrm{CM}$ bound; however, we have verified that for the optimal approach the fidelity reaches the CM regime very fast (for $N \gtrsim 12$ ). Therefore, CM do not provide a significant improvement over local measurements.

The estimation procedure goes as follows. After the $N$ measurements (one on each copy) have been performed, a list of outcomes is obtained, which we symbolically denote by $x$. Based on $x$, an estimate for $|\psi\rangle$ can be guessed, $\left|\psi_{\text {guess }}(x)\right\rangle$. The fidelity is then defined as the overlap

$$
\left|\left\langle\psi \mid \psi_{\text {guess }}(x)\right\rangle\right|^{2}=\frac{1+\vec{n} \cdot \vec{M}(x)}{2} \equiv f_{n}(x),
$$

where, as above, $\vec{n}$ is the unit vector on the Bloch sphere corresponding to the state $|\psi\rangle$ and $\vec{M}(x)$ is that corresponding to $\left|\psi_{\text {guess }}(x)\right\rangle$. The average fidelity can be written as $F \equiv\langle f\rangle=\sum_{x} \int d n f_{n}(x) P_{n}(x)$, where $P_{n}(x)$ is the probability of getting the outcome $x$ if the Bloch vector is $\vec{n}$, and $d n$ is the measure on the sphere in $3 \mathrm{D}$ (on the unit circle in 2D). Note that in both situations we have assumed no further prior knowledge of $|\psi\rangle$. 
Any local von Neumann measurement is represented by two projectors $O( \pm \vec{m})=(1 \pm \vec{m} \cdot \vec{\sigma}) / 2$, where $\vec{m}$ is a unit Bloch vector characterizing the measurement (in a spin system, e.g., $\vec{m}$ is the orientation of a Stern-Gerlach). The set of outcomes $x$ can be expressed as an $N$-digit binary number $x=i_{N} i_{N-1} \cdots i_{2} i_{1}$, where $i_{k}(=0,1)$ indicates that, upon measuring on the $k$ th copy, this is projected on the $O\left[(-)^{i_{k}} \vec{m}_{k}\right]$ projection space. The most general local measurement is realized when we allow $\vec{m}_{k+1}$ to depend also on the list of previous outcomes $i_{k} i_{k-1} \cdots i_{2} i_{1} \equiv x_{k}$ (hence, $x=x_{N}$ ). We thus write $\vec{m}\left(x_{k}\right)$ instead of $\vec{m}_{k}$. Note that $\vec{m}\left(x_{k}\right)$ satisfy

$$
\vec{m}\left(1 x_{k-1}\right)=-\vec{m}\left(0 x_{k-1}\right) .
$$

The fidelity of a general LOCC scheme can be written as

$$
F=\sum_{x=00 \cdots 0}^{2^{N}-1} \int d n \frac{1+\vec{n} \cdot \vec{M}(x)}{2} \prod_{k=1}^{N} \frac{1+\vec{n} \cdot \vec{m}\left(x_{k}\right)}{2},
$$

where the last product is the probability $P_{n}(x)$.

The optimal LOCC scheme is the one that maximizes (3) over the Bloch vectors $\vec{m}\left(x_{k}\right)$ and $\vec{M}(x)$. Using the Schwartz inequality, it is straightforward to see that the best guess $\vec{M}(x)$ must be proportional to the vector

$$
\vec{V}(x)=\int d n \vec{n} \prod_{k=1}^{N} \frac{1+\vec{n} \cdot \vec{m}\left(x_{k}\right)}{2},
$$

i.e., $\vec{M}(x)=\vec{V}(x) /|\vec{V}(x)|$. In this case, the maximum fidelity reads

$$
F=\frac{1}{2}\left(1+\sum_{x}|\vec{V}(x)|\right) .
$$

For a fixed set of measurements $\left\{m\left(x_{k}\right)\right\}$ (optimal or not) and a given set of outcomes $x$, the guess (4) provides the best estimate of the signal state. This simple and general result does not seem to be conveyed in the literature. We next show how (4) can be used to improve the quality of state estimation schemes based on local measurements.

Consider $N=2 \mathcal{N}$ copies of the state $|\psi\rangle$ whose vector $\vec{n}$ is known to be on the equator ( $x y$ plane) of the Bloch sphere (2D case). Since the expectation value of $\vec{\sigma}$ is $\langle\psi|\vec{\sigma}| \psi\rangle=\vec{n}$, the central limit theorem strongly suggests to adopt the following scheme. Let $\vec{e}_{1}, \vec{e}_{2}$ be the two unit vectors pointing along the $x$ and $y$ axes, respectively. For each $i(=1,2)$, perform $\mathcal{N}$ measurements of the observable $\sigma_{i}=O\left(\vec{e}_{i}\right)-O\left(-\vec{e}_{i}\right)$. Assume we have obtained $\mathcal{N} \alpha_{i}$ times the outcome +1 [consequently, $\mathcal{N}\left(1-\alpha_{i}\right)$ times the outcome -1]. One is driven to propose $\vec{M} \propto$ $\sum_{i} \alpha_{i} \vec{e}_{i}+\sum_{i}\left(1-\alpha_{i}\right)\left(-\vec{e}_{i}\right)$ (the mean value of these outcomes) as the Bloch vector of $|\psi\rangle$. More precisely,

$$
M_{i}(\alpha)=\frac{2 \alpha_{i}-1}{\sqrt{\sum_{j}\left(2 \alpha_{j}-1\right)^{2}}} .
$$

By doing so, the limiting behavior $M \stackrel{\mathcal{N} \rightarrow \infty}{\longrightarrow}\langle\psi|\vec{\sigma}| \psi\rangle=\vec{n}$ is ensured by the central limit theorem. With the techniques described below, one can show that the fidelity for the guess (6) has the asymptotic expression

$$
F_{\mathrm{CL}}=1-\frac{3}{8} \frac{1}{N}+\ldots
$$

where CL stands for central limit.

According to (4), however, this guess cannot be optimal. Let us show that indeed this scheme can be improved using (4) instead of (6). In this particular situation, Eqs. (5) and (4) read $F_{\mathrm{OG}}=\left(1+\sum_{\alpha}|\vec{V}(\alpha)|\right) / 2$ (OG stands for optimal guess) and

$$
\begin{aligned}
\vec{V}(\alpha)= & \prod_{i=1}^{2}\left(\begin{array}{c}
\mathcal{N} \\
\mathcal{N} \alpha_{i}
\end{array}\right) \int d n \vec{n} \\
& \times \prod_{j=1}^{2}\left(\frac{1+n_{j}}{2}\right)^{\mathcal{N} \alpha_{j}}\left(\frac{1-n_{j}}{2}\right)^{\mathcal{N}\left(1-\alpha_{j}\right)},
\end{aligned}
$$

where we have used the shorthand notation $\alpha=\left(\alpha_{1}, \alpha_{2}\right)$. To obtain the large $N$ limit of $F_{\mathrm{OG}}$, we use the formula

$$
\left(\begin{array}{c}
\mathcal{N} \\
\alpha \mathcal{N}
\end{array}\right) q^{\alpha \mathcal{N}}(1-q)^{(1-\alpha) \mathcal{N}} \rightarrow \frac{1}{\sqrt{2 \pi \mathcal{N} q(1-q)}} \times \exp \left(-\frac{\mathcal{N}}{2} \frac{(\alpha-q)^{2}}{q(1-q)}\right)\{1+O(1 / \sqrt{\mathcal{N}})\}
$$

with $q=\left(1+n_{j}\right) / 2$, and approximate the sum over the outcomes $\alpha$ by an integral. Performing the change of variables $r_{i}=2 \alpha_{i}-1$, we get

$$
\sum_{\alpha}|\vec{V}(\alpha)| \simeq \int d^{2} r|\vec{V}| \equiv \int d^{2} r\left|\int d n \vec{Q} e^{-(\mathcal{N} / 2) E}\right|
$$

with $E=\sum_{i=1}^{2}\left(n_{i}-r_{i}\right)^{2} /\left(1-n_{i}^{2}\right) \quad$ and $\quad \vec{Q}=P \vec{n}=$ $(\mathcal{N} / 2 \pi) \prod_{i=1}^{2}\left(1-n_{i}^{2}\right)^{-1 / 2} \vec{n}$. As it will become clear below, the terms of order $1 / \sqrt{\mathcal{N}}$ neglected in these approximations give no contribution to the final answer. The integral (10) will now be evaluated with the aid of the saddle point technique. We use spherical coordinates and write $\vec{n}=(\cos \phi, \sin \phi), \vec{r}=r(\cos \gamma$, $\sin \gamma)$. For large $\mathcal{N}$, the integrand peaks at $r=1$, which suggests writing $r=1+\xi$. We denote by $\phi_{m}$ or, equivalently, by $\vec{n}_{m}$, the location of the minimum of $E$, which can be computed as a power series in $\xi$ of the form $\phi_{m}=\gamma+\lambda_{1} \xi+$ $\lambda_{2} \xi^{2}+\ldots$ We next expand $E$ and $\vec{Q}$ as a power series in $t \equiv \vec{\phi}-\phi_{m}$ and write $\vec{Q}=\vec{L}+\vec{M} t+\vec{R} t^{2}+\ldots$ The exponential in (10) is seen to have the form

$$
e^{-(\mathcal{N} / 2) E}=e^{-(\mathcal{N} / 2) A} e^{-(\mathcal{N} / 2) B t^{2}} e^{-(\mathcal{N} / 2) \times O\left(t^{3}\right)},
$$

where $2 B$ is the second derivative of $E$ with respect to $t$, and the last exponential factor can also be expanded in 
powers of $t$. Neglecting contributions which vanish exponentially in $\mathcal{N}$, one has

$$
\vec{V}=\int d n \vec{Q} e^{-(\mathcal{N} / 2) E}=\frac{e^{(-\mathcal{N} / 2) A}}{\sqrt{2 \pi \mathcal{N} B}}\left(\vec{L}+\frac{\vec{R}}{\mathcal{N} B}+\ldots\right)
$$

where $d n=d \phi /(2 \pi)=d t /(2 \pi)$. At this point, all the coefficients can be expanded in powers of $\xi$, e.g., $B=$ $B_{0}+B_{1} \xi+\ldots, R=R_{0}+R_{1} \xi+\ldots$, and so on. Note, however, that the expansion of $A$ starts at order $\xi^{2}$, i.e., $\quad A=A_{2} \xi^{2}+A_{3} \xi^{3}+\ldots$ For $\vec{Q}=\vec{n} P=\vec{n}_{m} P+$ $\left(\vec{n}-\vec{n}_{m}\right) P$, we readily note that (12) yields

$$
\vec{V}=\vec{n}_{m} \int d n P e^{-(\mathcal{N} / 2) E}+\frac{\vec{I}}{\mathcal{N}}
$$

where $\vec{I} / \mathcal{N}$ is given by (12) with $\vec{Q}=\left(\vec{n}-\vec{n}_{m}\right) P$. We have written a factor $1 / \mathcal{N}$ in the last term to make explicit that this contribution is of order $\mathcal{N}^{-1}$. Hence,

$$
|\vec{V}|=\int d n P e^{-(\mathcal{N} / 2) E}+\frac{\vec{n}_{m} \cdot \vec{I}}{\mathcal{N}}+O\left(\frac{1}{\mathcal{N}^{2}}\right)
$$

and we finally have up to $O\left(1 / \mathcal{N}^{2}\right)$,

$$
\int d^{2} r|\vec{V}|=1-\int d^{2} r \int d n P\left(1-\vec{n} \cdot \vec{n}_{m}\right) e^{-(\mathcal{N} / 2) E} .
$$

The leading term (unity) comes from the first integral in (14), which is straightforward to compute exchanging the order of integration. Note also that the integral of the leading term on the right-hand side of (9), $\int d^{2} r P \exp (-\mathcal{N} E / 2)$, is one, and so is the sum over $\alpha$ on the left-hand side independently of $\mathcal{N}$. This shows that the terms of order $1 / \sqrt{\mathcal{N}}$ neglected in (10) indeed cancel. The subleading term in (15) can be computed using the general expression (12) where now $Q=\left(1-\vec{n} \cdot \vec{n}_{m}\right) P=$ $R t^{2}+O\left(t^{3}\right)$ (i.e., $L=M=0$ ). Moreover, only the leading terms in powers of $\xi$ have to be retained, namely, $A_{2}, B_{0}$, and $R_{0}$ (in particular, we just need $\lambda_{1}$ ). This is so because effectively $O\left(t^{2}\right)=O\left(\xi^{2}\right)=O(1 / \mathcal{N})$. The integral in (15) is then

$$
\int_{0}^{2 \pi} \frac{d \gamma R_{0}}{\sqrt{2 \pi \mathcal{N}^{3} B_{0}^{3}}} \int_{0}^{\infty} d r r \exp \left\{-\frac{\mathcal{N} A_{2}}{2}(r-1)^{2}\right\}=\frac{2 \pi}{\mathcal{N}} \int_{0}^{2 \pi} \frac{d \gamma}{2 \pi} \frac{R_{0}}{\mathcal{N} \sqrt{A_{2} B_{0}^{3}}},
$$

where again terms vanishing exponentially as $\mathcal{N} \rightarrow \infty$ have been neglected. The coefficient $\lambda_{1}$ is computed to be $\lambda_{1}=-\cot 2 \gamma$. With this, $B_{0}=2, R_{0}=(\mathcal{N} / 2 \pi) \csc 2 \gamma$, and $A_{2}=2 \csc ^{2} 2 \gamma$. The subleading term in (15) yields $-1 /(4 \mathcal{N})$, and substituting back in (5), we finally obtain

$$
F_{\mathrm{OG}}=1-\frac{1}{4} \frac{1}{N}+\ldots
$$

(recall that $N=2 \mathcal{N}$ ). As announced, this fidelity is larger than $F_{\mathrm{CL}}$. Moreover, it saturates the absolute upper bound given by $\mathrm{CM}$ even though classical communication has not been used. In this sense, the behavior of the most basic local scheme (without classical communication) in $2 \mathrm{D}$ is qualitatively similar to that obtained from CM provided the optimal guess (4) is used. Working along the same line, we have computed the asymptotic fidelities $F_{\mathrm{CL}}$ and $F_{\mathrm{OG}}$ in $3 \mathrm{D}$. One has

$$
F_{\mathrm{CL}}=1-\frac{6}{5} \frac{1}{N}+\cdots ; \quad F_{\mathrm{OG}}=1-\frac{13}{12} \frac{1}{N}+\cdots,
$$

where again we note that $F_{\mathrm{OG}}>F_{\mathrm{CL}}$. Despite this improvement, the CM bound $F_{\mathrm{CM}}=1-1 / N$ is not saturated, although the subleading term of $F_{\mathrm{OG}}$ is only $8 \%$ less than that of $F_{\mathrm{CM}}$.

Thus far, classical communication has not been exploited; i.e., the Bloch vectors $\vec{m}\left(x_{k}\right)$ were both $x$ independent and nonoptimal. We now turn to the full-fleshed LOCC schemes. Hereafter, only the general case 3D will be considered.
The first nontrivial case is $N=2$. Here, $x$ takes four possible values, $00,01,10$, and 11 . There are three independent vectors, namely, $\vec{m}(0), \vec{m}(00), \vec{m}(01)$ [the other three are obtained using Eq. (2)]. The first vector, $\vec{m}(0)$, is arbitrary and we take $\vec{m}(0)=\vec{z}$. The optimal fidelity is obtained by maximizing (5) with respect to $\vec{m}(00)$ and $\vec{m}(01)$. A straightforward calculation gives

$$
\sum_{x}|\vec{V}(x)|=\frac{1}{6} \sum_{k=0,1}\left(\left|\sin \frac{\theta_{0 k}}{2}\right|+\left|\cos \frac{\theta_{0 k}}{2}\right|\right),
$$

where $\theta_{x}$ is the polar angle of the vector $\vec{m}(x)$. The maximal value of (19) is attained for $\theta_{00}=\theta_{01}=\pi / 2$. Notice that $\theta_{00}$ and $\theta_{01}$ are maximized independently, so $\vec{m}(00), \vec{m}(01)$ do not need to be equal; they are required only to be orthogonal to $\vec{m}(0)$. Substituting back in (5), we find $F^{(2)}=(3+\sqrt{2}) / 6$ (see also [8]). From Eq. (4), we obtain the optimal guess

$$
M^{(2)}(x)=\frac{\vec{m}\left(x_{2}\right)+\vec{m}\left(x_{1}\right)}{\sqrt{2}}=\vec{s}(x),
$$

hence, e.g., $\quad \vec{M}^{(2)}(01)=[\vec{m}(01)+\vec{m}(1)] / \sqrt{2}=$ $[\vec{m}(01)-\vec{m}(0)] / \sqrt{2}$.

The case $N=3$ is very similar. The optimal Bloch vectors, $\vec{m}\left(x_{1}\right), \vec{m}\left(x_{2}\right), \vec{m}\left(x_{3}\right)$, are found to be mutually orthogonal. Since there is no further constraint, one can choose the three fixed (i.e., independent of $x$ ) vectors $\vec{m}\left(x_{k}\right)=\vec{e}_{k}, k=1,2,3$. This shows that for $N=3$ (as well as for $N=2$ ) the optimal estimation schemes based on local measurements do not require classical 
communication. For each outcome $x$, the optimal guess is $\vec{M}^{(3)}(x)=\left[\vec{m}\left(x_{3}\right)+\vec{m}\left(x_{2}\right)+\vec{m}\left(x_{1}\right)\right] / \sqrt{3}$, which is a straightforward generalization of $\vec{M}^{(2)}(x)$, and yields $F^{(3)}=(3+\sqrt{3}) / 6$. These results could somehow be anticipated: If $O(\vec{m})|\psi\rangle=0$, we can only be sure that the Bloch vector of $|\psi\rangle$ is not $-\vec{m}$. Intuition suggests to use the subsequent copies of $|\psi\rangle$ to explore the plane orthogonal to $\vec{m}$. Thus, the optimal Bloch vectors $\vec{m}\left(x_{k}\right)$ tend to be mutually orthogonal.

The case $N=4$ is more complex, since four mutually orthogonal vectors cannot fit onto the Bloch sphere. The solution exhibits some interesting features. First, the optimal Bloch vectors now depend on the outcomes of the previous measurements. Therefore, classical communication does play a crucial role for $N>3$. However, $\vec{m}\left(x_{1}\right) \perp$ $\vec{m}\left(x_{2}\right)$ and, as before, one can choose $\vec{m}\left(x_{i}\right)=\vec{e}_{i}$, for $i=1,2$. For only the third and fourth measurements, one really has to take different choices in accordance to the sequence of the preceding outcomes. The Bloch vectors of the third measurement can be parametrized by a single angle $\alpha$ as $\vec{m}\left(x_{3}\right)=\cos \alpha \vec{u}_{1}(x)+\sin \alpha \vec{v}_{1}(x)$, where $\vec{u}_{1}(x)=\vec{m}\left(x_{1}\right) \times \vec{m}\left(x_{2}\right)$ and $\vec{v}_{1}(x)=\vec{u}_{1}(x) \times \vec{s}(x)$ and $\vec{s}(x)$ is defined in (20). The optimal value of this angle is $\alpha=0.502$. We cannot give any insight as to why this value is optimal. However, in agreement with our intuition, $\vec{m}\left(x_{3}\right) \perp \vec{s}(x)$; i.e., the third measurement probes the plane orthogonal to the Bloch vector one would guess from the first two outcomes. Two angles are required to parametrize the vectors of the fourth measurement. They are given by $\vec{m}\left(x_{4}\right)=\cos \gamma \vec{u}_{2}(x)+\sin \gamma \vec{v}_{2}(x)$, where $\vec{u}_{2}(x)=\vec{s}(x) \times \vec{m}\left(x_{3}\right), \quad \vec{v}_{2}(x)=\cos \beta \vec{m}\left(x_{3}\right)-\sin \beta \vec{s}(x)$. The optimal values of these angles are $\beta=0.584, \gamma=$ 0.538 , and the corresponding fidelity is $F^{(4)}=0.8206$. This is just $1.5 \%$ lower than the absolute bound $F_{\mathrm{CM}}^{(4)}=$ $5 / 6=0.8333$ attained with CM. We also give the values of the maximal LOCC fidelities for $N=5,6$. They are $F^{(5)}=0.8450$ and $F^{(6)}=0.8637$. It is interesting to note that, for $N>3$, it pays to relax optimality at each step. Hence, one-step adaptive schemes [8,9] are not optimal, though the differences are very small; e.g., for $N=4$, $F^{(4)}>F_{\text {adaptive }}^{(4)}=(15+\sqrt{91}) / 30 \approx 0.8180$.

Having learned from the low $N$ cases, we are in the right position to compute the asymptotic fidelity of this scheme. For that, we take inspiration in variational methods as follows. Suppose we have performed a large number $N_{0}=\sqrt{N}$ of measurements and obtained the guess $\vec{M}_{0}$. It is clear that the subsequent $2 \bar{N}=N-N_{0}$ guesses will hardly differ from $\vec{M}_{0}$. We, hence, substitute in (3) the ansatz $\vec{M}(x) \approx \vec{M}_{0} \cos \omega+$ $\sin \omega(\vec{u} \cos \tau+\vec{v} \sin \tau)$, where $\vec{u}, \vec{v}$ are two unit vectors which along with $\vec{M}_{0}$ form an orthogonal bases, $\omega=\lambda$ $\sqrt{\left(2 \alpha_{u}-1\right)^{2}+\left(2 \alpha_{v}-1\right)^{2}}, \tan \tau=\left(2 \alpha_{v}-1\right) /\left(2 \alpha_{u}-1\right)$, and $\lambda$ is a variational parameter. As above, $\bar{N} \alpha_{u}\left(\bar{N} \alpha_{v}\right)$ is the number of times we obtain the outcome +1 when we measure $\vec{\sigma} \cdot \vec{u}(\vec{\sigma} \cdot \vec{v})$. Note that on average $\omega$ will be small since we expect $\alpha_{u, v} \approx 1 / 2$, and we need to retain terms up to order $\omega^{2}$. Putting all this together, one gets from (3)

$$
F \geq 1-(1-\lambda)^{2}\left(1-F_{0}\right)-\frac{\lambda^{2}}{N-N_{0}}+\cdots,
$$

where $F_{0}$ is the optimal fidelity for $N_{0}$ measurements and the dots stand for subleading terms in inverse powers of $N$ and $N_{0}$. We readily see that the optimal choice is $\lambda=1$, which leads to $F \approx 1-1 / N$ [12]. Hence, our LOCC scheme does saturate the CM bound. Furthermore, numerical analysis reveals that the $\mathrm{CM}$ regime is reached for values of $N$ as low as 12 .

In summary, we have obtained the optimal LOCC estimating scheme for general qubit (pure) states and shown that its fidelity saturates the collective measurement bound. For states that are known to lay on a meridian of the Bloch sphere (2D case), we have explicitly given a scheme whose fidelity saturates this bound without invoking classical communication.

We acknowledge financial support from CIRIT Project No. SGR-00185, Spanish Ministry of Science and Technology Project No. BFM2002-02588, and the European Funds for Regional Development (FEDER).

[1] A. Peres and W. K. Wootters, Phys. Rev. Lett. 66, 1119 (1991).

[2] S. Massar and S. Popescu, Phys. Rev. Lett. 74, 1259 (1995).

[3] K. R. Jones, Phys. Rev. A 50, 3682 (1994); D. Brody and B. Meister, Phys. Rev. Lett. 76, 1 (1996); Z. Hradil et al., Phys. Rev. A 62, 014101 (2000); S. Massar, Phys. Rev. A 62, 040101(R) (2001); K. Banaszek and I. Devetak, Phys. Rev. A 64, 052307 (2001).

[4] E. Bagan et al., Phys. Rev. Lett. 85, 5230 (2000); Phys. Rev. A 63, 052309 (2001); A. Peres and P. F. Scudo, Phys. Rev. Lett. 86, 4160 (2001); E. Bagan, M. Baig, and R. Munoz-Tapia, Phys. Rev. A 64, 022305 (2001).

[5] R. D. Gill and S. Massar, Phys. Rev. A 61, 042312 (2000).

[6] K. Matsumoto, J. Phys. A 35, 3111 (2002).

[7] E. Bagan et al. (to be published).

[8] D. G. Fisher, S. H. Kienle, and M. Freyberger, Phys. Rev. A 61, 032306 (2000).

[9] Th. Hannemann et al., Phys. Rev. A 65, 050303(R) (2002).

[10] A.S. Holevo, Probabilistic and Statistical Aspects of Quantum Theory (North-Holland, Amsterdam, 1982).

[11] R. Derka, V. Buzek, and A. K. Ekert, Phys. Rev. Lett. 80, 1571 (1998).

[12] An alternative proof of this asymptotic limit based on the quantum Cramer-Rao bound and one-step adaptive scheme can be found in [5] (see also [6]). 